Revue d'histoire de l'Amérique française

REVUE D.HISTOIRE DE L'AMÉRIQUE FRANÇAISE

\title{
La grève des charretiers à Montréal, 1864
}

\section{Margaret Heap}

Volume 31, numéro 3, décembre 1977

URI : https://id.erudit.org/iderudit/303634ar

DOI : https://doi.org/10.7202/303634ar

Aller au sommaire du numéro

Éditeur(s)

Institut d'histoire de l'Amérique française

ISSN

0035-2357 (imprimé)

1492-1383 (numérique)

Découvrir la revue

Citer cet article

Heap, M. (1977). La grève des charretiers à Montréal, 1864. Revue d'histoire de l'Amérique française, 31(3), 371-395. https://doi.org/10.7202/303634ar d'utilisation que vous pouvez consulter en ligne.

https://apropos.erudit.org/fr/usagers/politique-dutilisation/ 


\title{
LA GRÈVE DES CHARRETIERS À MONTRÉAL, 1864*
}

\author{
MARGARET HEAP \\ Groupe de recherche sur la \\ société montréalaise au $19^{e}$ siècle \\ Département d'bistoire \\ Université du Québec à Montréal
}

En septembre 1864 éclate une grève des charretiers de Montréal qui paralyse le port et la vie commerciale de la ville. Elle devient la préoccupation centrale des marchands et des journaux pendant plusieurs jours, provoque l'intervention du maire et du conseil municipal - bref, bouleverse la ville pendant presqu'une semaine. Les résultats de la grève consacreront les débuts d'une nouvelle ère dans le voiturage à Montréal et confirmeront l'émergence d'un nouveau rapport de forces; ils accentueront la tendance à l'établissement de rapports et de conditions de travail qui privent les charretiers de leurs moyens de travail et conduisent à leur prolétarisation.

Pour bien situer cette grève et mieux en comprendre les enjeux, examinons d'abord brièvement la place et le rôle qu'occupent le transport et plus particulièrement, le voiturage au début des années 1860 à Montréal.

\section{Le voiturage}

Le secteur du transport joue un rôle-clé dans l'économie canadienne au $19^{\mathrm{e}}$ siècle. Dans un pays dont l'économie est axée depuis longtemps sur l'exportation de quelques produits de base (fourrures, bois, blé), le développement d'un réseau adéquat de transports demeure une préoccupation centrale de la bourgeoisie commerciale. Les débuts de l'industrialisation ne font qu'accentuer cette importance, ainsi que la tendance vers la concentration qui s'y manifeste.

* Version révisée d'une communication présentée au congrès de l'Institut d'histoire de l'Amérique française, à Rimouski, en septembre 1977.

[371]

RHAF, vol. 31, n 3 (décembre 1977) 
De la même façon, le voiturage joue un rôle fondamental dans l'économie de Montréal au $19^{\mathrm{e}}$ siècle. Métropole commerciale, tête de navigation, Montréal est le point principal de transbordement, tant pour les marchandises remontant le fleuve vers l'intérieur que pour les produits d'exportation (le bois équarri excepté). Toutes ces marchandises doivent être transportées entre divers points dans la ville - quais, entrepôts, canal - et ce sont les charretiers qui assurent cette circulation.

Les travailleurs des transports - les charretiers, les débardeurs, les ouvriers des chemins de fer - constituent d'ailleurs un des groupes de travailleurs les mieux organisés et les plus combatifs à cette époque, comme en témoignent les conflits de travail et l'émergence de plusieurs organisations ouvrières importantes ${ }^{1}$. Le nombre important de grèves qui se produisent parmi ces ouvriers dans la deuxième moitié du $19 \mathrm{e}$ siècle ${ }^{2}$ peut étonner à première vue. Mis à part les ateliers des chemins de fer, les secteurs touchés sont caractérisés par le travail saisonnier, demandant peu ou pas de qualifications, et accompli fréquemment de façon isolé ou en petits groupes $^{3}$. Or, ces conditions sont souvent des obstacles au regroupement et à l'organisation des ouvriers. Cependant, malgré cela, ces ouvriers possèdent une certaine force, et l'impact de leurs grèves - y compris celles des charretiers - reflète le rôle important qu'ils jouent dans l'économie, et surtout dans les échanges commerciaux. Et ces ouvriers ont des raisons pressantes de se regrouper et réagir, car leurs conditions de vie et de travail sont bouleversées, tout comme le sont celles des ouvriers des manufactures, par le capitalisme industriel naissant.

1 Le Quebec Ship Labourers' Benevolent Society en est sans doute l'exemple le plus remarquable; voir J. I. Cooper, "The Quebec Ship Labourers' Benevolent Society», CHR, 30, no 4 (décembre 1949): 336-343. Voir aussi les témoignages des débardeurs de Québec devant la Commission royale d'enquête sur les rapports qui existent entre le capital et le travail au Canada, (Québec) 2: 741-757; 1078-1090; 1132-33; aussi le témoignage de Ray, qui présente diverses clauses des règlements de la Society: 1021-1022. On pourrait aussi mentionner les syndicats des ouvriers des chemins de fer, ainsi que les assemblées de débardeurs et de charretiers au sein des Chevaliers du travail; voir Noël Bélanger et al, Les travailleurs québécois 1851-1896, (Montréal, PUQ, 1973), 214-216.

2 Jean Hamelin, Paul Larocque et Jacques Rouillard, Répertoire des grèves dans la province de Québec au XIX" siècle (Montréal, Les Presses de l'École des Hautes Études commerciales, 1970).

3 Voir les témoignages devant la Commission royale d'enquête, op. cit.; aussi ceux des débardeurs à Montréal et des employés des chemins de fer. 
Dans le cas des chemins de fer, ces transformations passent par la création «de toute pièce» d'une nouvelle technologie et souvent même par le recours à une nouvelle main-d'œuvre. Pour les débardeurs elles se concrétisent dans une certaine spécialisation et une certaine mécanisation du travail ${ }^{4}$. Dans le cas des charretiers les transformations sont, par contre, plus subtiles. Ce ne sont ni leurs outils, ni leurs «moyens de travail», ni même la division, l'organisation physique du travail qui seront transformés de façon marquée au $19^{\mathrm{e}}$ siècle, mais plutôt le rapport entre les charretiers et leurs outils, leurs rapports de travail.

Plus particulièrement, les charretiers montréalais sont eux aussi touchés, et leurs conditions de travail transformées. Bien qu'il y ait amélioration des voitures (capacité accrue) et de la qualité des chevaux, l'évolution technologique qui bouleverse d'autres secteurs du transport ne transformera pas pour l'essentiel le transport intraurbain des marchandises avant la fin du siècle ${ }^{5}$. Cependant, les transformations dans les autres moyens de transport qui constituent les liens externes de la ville n'en marquent pas moins l'industrie du voiturage à Montréal, et ceci, de deux façons contradictoires. D'une part, elles rétrécissent en partie le territoire couvert par le charroyage, éliminant à toujours certains trajets des charretiers; mais d'autre part, l'amélioration des conditions de circulation de marchandises et l'augmentation du volume de commerce créent aussi de nouvelles possibilités de travail pour les charretiers. Ainsi, l'ouverture du canal Lachine en 1825 fait disparaître la nécessité de tout faire transporter par wagon de Montréal à Lachine. Certains craignent même pour l'avenir des charretiers. Mais les effets néfastes sont apparemment bientôt atténués par le volume accru de trafic dans le port de Montréal ${ }^{6}$. De la même façon, l'arrivée du GrandTronc a des effets contradictoires: dans un premier temps, l'ouverture du chemin de fer avant que le pont Victoria ne soit achevé permet aux charretiers, semble-t-il, de réaliser des affaires d'or penop. cit.

4 Voir encore les témoignages devant la Commission royale d'enquête,

5 D'autres moyens de transbordement, comme, par exemple, des chalands, semblent peu utilisés; voir le Montreal Herald (29 septembre, 1864), 1.

6 Certains entrepreneurs commencent même à organiser des services de charroyage qu'ils offrent aux marchands en même temps que des services d'entreposage; voir l'annonce de Thomas Porteous, Montreal Herald (21 mai 1825). 
dant les mois d'hiver ${ }^{7}$; la localisation du premier dépôt à Pointe Saint-Charles implique un charroyage important entre celui-ci et les entrepôts et quais du centre-ville; mais le progrès subséquent - le dépôt Bonaventure, les projets d'embranchements sur les quais soulève la menace d'un rétrécissement additionnel du domaine du voiturage. Cependant, dans la mesure où le chemin de fer contribue à augmenter la circulation totale de marchandises dans la ville, le voiturage en tire profit $^{8}$.

Le nombre de charretiers croît avec la population et l'économie de la ville. De 201 charretiers en $1825^{\circ}$, on passe à 1188 en $1861^{10}$. Il est également possible qu'un nombre indéterminé de ceux qui travaillent effectivement comme charretiers s'identifie plutôt comme journaliers, mais nous n'avons pas pu vérifier cette hypothèse. Cependant, dans les décennies 1860 et 1870 , le nombre de voitures et voitures de louage semble augmenter plus vite que le nombre de charretiers ${ }^{11}$. S'il y a alors une augmentation parallèle du nombre de gens travaillant comme charretiers, il s'agit sans doute de char-

7 Jean-Claude Robert, Montréal 1821-1871, Aspects de l'urbanisation (Thèse de doctorat, École des Hautes Études en sciences sociales, 1977), 273; et Kathleen Jenkins, Montreal, Island City of the St. Lawrence (New York, Doubleday \& Co., 1966), 353-354.

8 Montreal Transcript (12 janvier 1856), 2; Montreal Gazette (3 octobre 1864), 2; Montreal Herald (1 octobre 1864), 2.

9 Jean-Paul Bernard, Paul-André Linteau et Jean-Claude Robert, «La structure professionnelle de Montréal en 1825», RHAF, 30, 3, (déc. 1976): 383-415.

10 Nombre que nous avons repéré dans le recensement manuscrit de 1861, et qui exclut une série de conducteurs plus spécialisés, tels les laitiers, les livreurs de pain ou de bière, etc. Les recensements de 1842 et 1851 fournissent aussi un relevé des charretiers à Montréal, mais nous croyons que ces recensements sousestiment de façon importante leur nombre. Ainsi, en 1842, il y aurait 276 charretiers, mais il ne s'agit que des charretiers qui sont aussi chefs de ménage; et en 1851, recensement qui donne les occupations de tous, on n'en dénombre que 250 , tandis que le rôle d'évaluation de la même année indique 257. Rappelons qu'en 1861, le rôle d'évaluation indique 675 charretiers, pour 1188 repérés dans le recensement, ce qui suggère l'ampleur de la sous-estimation en 1851. APC, Recensement de 1842, listes nominatives; APC, Recensement des Canadas, 1860-61, listes nominatives; Recensement de 1851-52, 1: tableau IV; Archives de la ville de Montréal, rôle d'évaluation, 1851 .

11 Voir, entre autres, le recensement de 1871 , où on dénombre 7766 véhicules de transport et le Rapport annuel du chef de police, 1876, où le nombre de permis pour des voitures a augmenté de façon marquée par rapport aux années 1860, tandis que le nombre de charretiers augmente beaucoup moins vite - à 1309 (cochers et charretiers) en 1871 et à 1633 (cochers et charretiers) en 1881, toujours à Montréal; Recensement de 1871, tableau XIII, et Recensement de 1881, tableau XIV. 
retiers ne possédant ni cheval, ni voiture, et travaillant peut-être comme employé régulier, salarié, pour des marchands ou des manufacturiers.

Malheureusement, notre connaissance de l'organisation du voiturage au début des années 1860 est encore fragmentaire. Nous croyons que le port constitue encore le pôle central du travail des charretiers, et nous en voyons la preuve dans les tarifs imposés par la municipalité. Celle-ci fixe des taux particuliers pour le transport des produits de base comme la farine, le blé, la potasse, le bois, etc., et pour les trajets autour du port et du canal. Ajoutons que l'unique poste public de charretiers de marchandises est situé au bord de l'eau ${ }^{12}$. Il faut aussi tenir compte du fait que les manufactures qui se développent de plus en plus dans la ville demandent des services de voiturage pour s'approvisionner en matières premières et en combustibles, et pour expédier les produits finis. Certains font appel au voiturage ${ }^{13}$, mais nous ne savons pas encore dans quelle mesure d'autres manufacturiers, et même certains marchands, disposent de leurs propres services de voiturage ${ }^{14}$. Finalement, les dépôts du Grand-Tronc constituent un autre centre de voiturage, d'une importance croissante pendant ces années.

En général, les charretiers eux-mêmes sont encore des petits artisans indépendants, dont les conditions de travail - les tarifs et les règlements auxquels ils sont soumis - sont déterminées par la municipalité ${ }^{15}$. On retrouve, bien sûr, une proportion importante soit entre $24 \%$ et $30 \%$ - des charretiers qui sont déjà prolétarisés, ne possédant ni cheval ni voiture, et qui doivent travailler pour

12 Compilation of the Bye-laws and Police Regulations in Force in the City of Montreal. With an Appendix Containing Extracts from Provincial Enactments (Montreal, James Starke \& Co., 1842), 68-69, et The Charter and By-Laws of the City of Montreal; together with Miscellaneous Acts of the Legislature relating to the City: with an appendix, compiled, revised and codified... by Chs. Glackmeyer (Montreal, John Lovell, 1865), 391-394.

13 Ainsi, Curran, un des leaders de la grève en 1864 , transporte du charbon pour une usine employant 200 personnes, L'Union Nationale (28 septembre 1864), 3 ; et Brown \& Childs, manufacture importante de bottes et chaussures, fera appel aux services de Shedden, Montreal-Gazette (14 décembre 1865), 1.

14 Nous savons, par exemple, que des manufacturiers et marchands prennent des permis pour 259 voitures en 1863 et 317 voitures en 1864, mais nous ne savons pas qui conduit ces voitures. Voir le Annual Report of the Chief of Police (1864), op. cit., et Annual Report of the Sub-Chief of Police (1865), op. cit.

is Compilation of the Bye-Laws..., op. cit., et The Charter and By-laws of the City of Montreal..., op. cit. 
d'autres charretiers, ou directement pour des marchands ou manufacturiers ${ }^{16}$.

Il y a aussi une petite minorité qui possède plusieurs chevaux et/ou voitures ${ }^{17}$ : 119 charretiers, ou $10 \%$. Certains de ces derniers s'identifient comme maitres-charretiers, mais ce titre ne semble pas avoir une signification nettement définie, et son utilisation varie beaucoup selon les sources consultées. Cependant, ceux que nous pourrions considérer comme de véritables maîtres-charretiers ceux, par exemple, qui possèdent cinq chevaux ou plus, ou qui ont une concentration importante de voitures par rapport au nombre de leurs chevaux ${ }^{18}$ - forment un groupe encore plus réduit: il n'y en a que 26 (2,2\% de tous les charretiers). Ces «maitres-charretiers» (peu importe qu'ils s'identifient comme tel ou non) contrôlent presque $16 \%$ des 1239 chevaux possédés par les charretiers, et presque $13 \%$ de leurs 1067 voitures de louage. Ils emploient évidemment d'autres charretiers, mais sur une échelle assez réduite pour la plupart. Ainsi, un Toussaint Lecompte, qui, avec 21 chevaux, emploie 12 hommes, est toujours une figure exceptionnelle ${ }^{19}$. $\lambda$ ces maîtrescharretiers s'ajoutent les «City Express Companies» qui commencent à se développer pendant cette période, dont celle de E. Bancroft, qui a huit chevaux, et celle de Charles Larin, qui semble la plus importante, avec 13 chevaux et 33 voitures de louage ${ }^{20}$.

La grande majorité des charretiers ne fait pas partie de ces deux groupes. Près de $50 \%$ d'entre eux possèdent au moins un cheval et une voiture; tandis qu'environ $20 \%$ ne possèdent que des chevaux. Dans ces deux cas, nous croyons qu'il s'agit de petits artisans indépendants, car rien n'indique que le manque de voitures signifie pour un charretier la perte de son indépendance: certains des maîtres-charretiers les plus importants (par exemple, Toussaint Lecompte) ne semblent pas posséder de voitures.

Ainsi, la prolétarisation et la concentration des moyens de travail au sein même de l'industrie du voiturage sont certes commen-

16 Ces chiffres, et ceux qui suivent, sont les résultats de nos compilations à partir des listes nominatives, recensement de 1861 .

17 Soit 3 chevaux ou plus, ou 4 voitures ou plus.

18 Nous parlons de «véritables maîtres-charretiers» ici, car il nous semble fort probable qu'un charretier possédant ce nombre de chevaux ou de voitures doit employer un ou plusieurs autres charretiers.

19 APC, recensement de 1861 , listes nominatives, folio 12438.

20 Pour E. Bancroft, voir le Rôle d'Évaluation de la ville de Montréal, 1860 , quartier Centre; pour Larin, APC, recensement de 1861, listes nominatives, folio 4560. 
cées, mais ne touchent qu'une minorité de charretiers, et le processus est lent et encore peu développé. La plupart des charretiers ont encore tout à perdre - leurs moyens de travail, leur indépendance.

Et ce sera l'enjeu fondamental de la grève de 1864 qui les oppose à la compagnie du chemin de fer du Grand-Tronc. Car, avec l'agence de voiturage formée par John Shedden, le Grand-Tronc accélère et télescope le développement capitaliste de l'industrie du voiturage. Shedden, tant par la diversité et l'étendue de ses opérations que par l'organisation et le fonctionnement de son agence de voiturage et par ses rapports privilégiés avec le Grand-Tronc, bouleverse l'industrie du voiturage à Montréal. Il menace la survie des petits charretiers indépendants, provoquant la résistance des charretiers et des maîtres-charretiers.

\section{L'implantation de Shedden à Montréal}

Né en Écosse, John Shedden (1829-1873) ${ }^{21}$ a été entrepreneur dans la construction de chemins de fer aux États-Unis avant de s'établir au Canada. En 1855 il a fondé, avec William Hendrie, une agence de voiturage qui devient l'agence exclusive de la Great Western Railway Company à Hamilton et à London. Avec l'ouverture du Grand-Tronc en 1856, ils proposaient d'étendre leur agence aux villes desservies par le Grand-Tronc entre Montréal et Toronto, et demandaient au Grand-Tronc de leur accorder des contrats exclusifs pour le voiturage du fret transporté par le chemin de fer dans ces villes; leur ambition était, selon les dires d'un contemporain, de devenir les «Pickfords of Canada» 22 . Leurs agences semblent avoir été établies dès 1856 à Toronto, et peu de temps après dans plusieurs autres villes, malgré une certaine opposition. Cependant, il fallut plusieurs années avant que leur système ne soit implanté à Montréal. La société Hendrie et Shedden s'est dissoute

21 Pour la vie et les travaux de John Shedden, voir Myles Pennington, Railways and Otherways (Toronto, Williamson \& Co., 1896), 104-109: Montreal Gazette (19 mai 1873); et W. Stewart Wallace, The Macmillan Dictionary of Canadian Biography (1963), 686.

22 Pickfords était une grande compagnie de voiturage interurbain qui avec l'arrivée des chemins de fer a su se recycler très rapidement dans la cueillette et la livraison de fret pour les compagnies de chemin de fer, jouissant souvent de conditions privilégiées. Voir Myles Pennington, op. cit., 104-105, et H. D. Dyos et D. H. Aldcroft, British Transport: An Economic Survey from the Seventeenth Century to the Twentieth (Penguin Books, 1974), 228. 
après quelques années, Hendrie continuant à s'occuper du voiturage relié au chemin de fer du Great Western, et Shedden prenant en main celui du Grand-Tronc.

Malgré les préférences évidentes d'au moins plusieurs cadres du Grand-Tronc, ce système a tardé à s'implanter à Montréal. Toutefois, il semble que le Grand-Tronc utilisait déjà au moins une, sinon plusieurs agences de voiturage, de préférence à une foule de charretiers individuels, et que les conditions et tarifs accordés étaient semblables à ceux obtenus par Shedden quelques années plus tard. Ainsi, en 1862, c'est Toussaint Lecompte, un des maîtres-charretiers les plus importants, qui détient cette agence ${ }^{23}$. Cependant, cette dernière ne constitue apparemment pas un monopole, comme ce sera le cas avec Shedden, et les tarifs de voiturage restent distincts des tarifs de fret du chemin de fer.

Mais le Grand-Tronc est convaincu de l'efficacité et des bénéfices du système d'agence exclusive proposé et établi par Shedden ailleurs, et veut l'introduire à Montréal, qui représente une partie très importante de son marché de fret. Les avantages sont expliqués par la Montreal Gazette, qui justifie cette décision ainsi:

...All the great railway companies in Britain, if we mistake not, certainly many of them, at their principal stations undertake the delivery of goods at the place of destination by their own carriers, i.e., those who contract with them to do their cartage. This is found necessary in order to expedite business. It has been found necessary by the Grand Trunk Railway Company to do this also at its principal stations in Canada. ... [The old system] involved the labour of more men than were needed, and the inconvenient crowding of freight yards very often when trade was brisk, involving serious cost and hindrance. This, we apprehend, has been the common experience of railway companies at all great depots. To keep the place clear, and handle the goods conveniently and cheaply, they need to direct the carting themselves. Therefore, the G.T.R. Company employed Mr. Shedden to do the work. ${ }^{24}$

Le Grand-Tronc cite aussi le problème de la responsabilité des marchandises endommagées entre les entrepôts des marchands et le dépôt du chemin de fer, les coûts de la manutention des mar1863).

23 Montreal Gazette (3 octobre 1864), 2; Montreal Herald (23 janvier

24 Montreal Gazette (30 septembre 1864). 
chandises arrivant au dépôt pour expédition par le chemin de fer, et le danger que les dépôts de la compagnie deviennent des salles d'exposition pour les marchands ${ }^{25}$.

Shedden s'établit donc en affaires à Montréal en janvier 1863:

The Subscriber having become CARTAGE AGENT of the GRAND TRUNK RAILWAY COMPANY, in the place of Mr. T. Lecompte, is prepared to Receive and Deliver all Property going from and coming to Montreal by the Grand Trunk Railway, as well as other Cartages which may be intrusted to him. ${ }^{26}$

Toussaint Lecompte annonce qu'il lui a cédé tous ses intérêts dans les affaires de voiturage ${ }^{27}$. Shedden obtient en outre du GrandTronc un monopole par la création d'une structure de tarifs uniques qui incluent aussi bien le transport ferroviaire que le voiturage entre les dépôts et la ville. Ainsi, même si un marchand ou un manufacturier préfère utiliser ses propres charretiers pour chercher ou faire livrer des marchandises à la gare, il doit payer le même tarif de fret que ceux qui utilisent les services de l'agent de voiturage du Grand-Tronc.

Les charretiers de Montréal ne tardent pas à réagir. Le 27 janvier, 22 d'entre eux, s'identifiant comme maîtres-charretiers, signent une annonce sous forme de lettre ouverte aux marchands montréalais. Cette lettre expose leurs griefs envers le nouveau système, et demande l'intervention des marchands en leur faveur, sans proposer, cependant, de moyens concrets pour le faire:

\section{GENTLEMEN :}

We the undersigned Master Carters of Montreal, beg respectfully to lay our grievances before you, trusting you will give a fair and impartial opinion in our favor.

25 Montreal Herald (1 ${ }^{\mathrm{er}}$ octobre 1864); Montreal Gazette (14 décembre 1865).

26 Montreal Herald (23 janvier 1863). Shedden est manifestement un soustraitant du Grand-Tronc. C'est grâce au Grand-Tronc que son agence a vu le jour à Montréal, et elle est protégée par et identifiée à cette compagnie: l'agence est connue comme le Grand Trunk Railway Cartage Office, les lettres G.T.R. paraissent sur ses voitures, et ses employés seront connus comme les «Grand Trunk Carters». Cependant, le Grand-Tronc peut toujours, au besoin, se défendre d'y avoir des intérêts, en prétendant qu'il s'agit simplement d'une agence sous contrat. De fait, tout est au nom de Shedden: les permis municipaux, les chevaux, les voitures. Montreal Herald (4 janvier 1864 et 30 avril 1864); Montreal Gazette (14 décembre 1865); Myles Pennington, op. cit., 105-106.

27 Montreal Herald (23 janvier 1863). 
The Grand Trunk Company have issued a circular stating that they will in a few days publish a tariff for the carting and carrying of all goods received at and forwarded from this city.

In the first place, you are aware that the present Staff at Pointe St. Charles are under the control of entire strangers to the mercantile necessities of this city, and that what they propose to do cannot be done, unless at the cost of the utter ruin of many poor and deserving men, long residents of this city and for the benefit of Mr. Sheddon (sic) of Toronto.

But it is too well known that, at the opening of the navigation, if you now allow this great monopoly to be carried out, you will find yourselves in a very awkward position, when you have no competition in the carting business.

$\cdots$

Show Mr. Brydges ${ }^{28}$ and his clique that they cannot govern the mercantile interests of this city, to the detriment of old, honest, faithful and tax-paying carters of Montreal.

Trusting that you will take the necessary steps to prevent this system from coming into operation, by such means as you deem necessary; while we, the Master-Carters, promise to do, as heretofore, all in our power to facilitate the business entrusted to us, and by honest means and fair play, to uphold the fame of the City of Montreal. ${ }^{29}$

Après cette première réaction des charretiers, on n'en retrouve guère d'autres avant l'automne 1863. Ceci s'explique en partie par les délais de mise en route de l'agence de Shedden ${ }^{30}$, et en partie par le fait qu'il y a encore bien assez de travail tant pour Shedden que pour les charretiers pendant les mois d'été, saison des activités portuaires. Cependant, la venue de l'hiver amène un net ralentissement des activités, et la menace posée par le monopole de Shedden se fait sentir de façon aiguë ${ }^{31}$. De plus, une grève déclenchée en septembre, comme ce sera le cas deux années de suite, est particulièrement efficace, à cause de la grande activité dans le port avant la fin de la navigation.

\section{La grève de l'automne 1863}

Le 10 septembre, la question du charroyage du Grand-Tronc est soulevée au conseil municipal. Un conseiller souligne que le

28 Brydges est le gérant du Grand-Tronc à Montréal à cette époque.

29 Montreal Herald (27 janvier 1863).

30 Témoignage de M. Stevenson, Montreal Gazette (14 décembre 1865).

31 Montreal Herald (1 ${ }^{\text {er }}$ octobre 1864); Montreal Witness (8 octobre 1864). 
nouveau système du Grand-Tronc fonctionne aux dépens des charretiers détenteurs de permis municipaux, dont un certain nombre ont même été forcés d'abandonner leurs affaires. Son intervention, cependant, ne débouche pas sur des propositions concrètes ${ }^{32}$. Samedi, le 19 septembre, les charretiers tiennent une réunion où ils décident de se mettre en grève pour obtenir un accord quant aux tarifs de charroyage vers les dépôts du chemin de $\mathrm{fer}^{33}$.

La grève est déclarée le lundi matin, et quelques charretiers sont arrêtés «pour avoir intimidé d'autres qui voulaient travailler ${ }^{34}$, mais le mouvement de grève se dissipe vite dans l'attente d'une intervention du Board of Trade qui nomme un comité spécial chargé de trouver un terrain d'entente entre les charretiers et le Grand-Tronc ${ }^{35}$. Les charretiers réclament, semble-t-il, une remise sur le tarif global de fret pour les marchands qui font transporter leurs marchandises par des charretiers autres que ceux de Shedden. La médiation du Board of Trade porte fruit, car quelques jours plus tard, Brydges, le gérant du Grand-Tronc à Montréal, annonce que sa compagnie a accepté d'accorder des remises de $\$ 0.25$ le tonneau pour des marchandises envoyées à la gare Bonaventure et $\$ 0.35$ le tonneau pour celles envoyées au dépôt de Pointe Saint-Charles par des marchands utilisant les charretiers de leur choix.

Cependant, le Grand-Tronc prend bien soin de souligner qu'il s'agit d'une mesure temporaire, d'une concession unilatérale, et qu'il ne se sent nullement lié par ces conditions:

For the present, therefore, but on the clear understanding that it is simply for a limited time, and involving no obligation whatever on the Company to continue it, unless they find it judicious to do so, I will agree temporarily to alter the deductions to be made from the rates, if goods are taken from the City to this Company's Stations by other carters than those who have a contract with this Company. ...

I trust you will accept this change as an evidence of the desire of the Company, and of myself, to do everything in our power to meet the wishes of the merchants of Montreal.

32 Montreal Herald (10 septembre 1863).

33 Montreal Herald (21 septembre 1863).

34 "for intimidating carters from working», Montreal Herald (22 septembre 1863).

35 APC, MG 28, III: 44. Montreal Board of Trade, Minute Book III : 306308, 312; Montreal Transcript (22 septembre 1863). 
It is right, at the same time, to say that we may not find it possible to continue the change which I now propose to make.

In that event it must be clearly understood that the Company will revert to the arrangements at present in existence. ... ${ }^{36}$

Le ton de sa lettre, ainsi que les commentaires des journaux, laissent croire que le Grand-Tronc visait, avant tout, à se concilier les marchands; que les charretiers se soient mis en grève ou que les nouveaux tarifs répondent au moins partiellement à leurs revendications semblent des aspects bien secondaires, aussi bien pour la compagnie que pour les journalistes...

We think the mercantile community are indebted to the Board of Trade for this successful intervention on their behalf; and we are glad also that something has been done for the large class of hard-working men hitherto engaged in the cartage business. ${ }^{37}$

Mr. Brydges' letter evidences a desire to accomodate the trade of Montreal, and to meet the wishes of the merchants. ${ }^{38}$

Avec cet accord, la question est mise en veilleuse un certain temps, et on entend peu parler des charretiers ou de Shedden.

Shedden, cependant, continue à consolider son agence. En avril 1864, on fait état des écuries - probablement les plus grandes de la ville, dit-on - qu'il fait construire dans le quartier Sainte-Anne, et qui pourront abriter 64 chevaux. On souligne aussi la haute qualité de ses chevaux ${ }^{39}$. En mai 1864, il obtient des permis pour sept voitures à un cheval et 17 voitures lourdes à deux chevaux; au cours de l'année il en ajoute d'autres ${ }^{40}$.

Il est évident que l'ampleur de ses opérations dépasse déjà de loin celles des charretiers les plus importants en $1861^{41}$. Nous ne savons pas combien de personnes - charretiers ou journaliers -

36 Montreal Transcript (28 septembre 1863).

Montreal Herald (28 septembre 1863).

Montreal Transcript (28 septembre 1863).

Montreal Herald (6 avril 1864).

40 Témoignages d'Olivier Bouchard, sergent de police, Montreal Gazette (14 décembre 1865).

41 Cet écart est encore plus important si on considère que le nombre de voitures donné dans le recensement inclut d'habitude et les voitures d'été et les voitures d'hiver, tandis que les permis obtenus de la municipalité sont valables pour deux voitures chacun — une voiture d'été et la voiture d'hiver correspondante. 
sont employées par Shedden. Certains marchands affirmeront plus tard qu'ils sont obligés de fournir leurs propres employés pour charger et décharger les wagons quand ils font appel aux services de Shedden (contrairement à ce qui arrive avec les charretiers indépendants) ${ }^{42}$, ce qui nous porte à croire que Shedden utilise un personnel réduit au minimum.

Cependant, en mai 1864, il emploie au moins 24 charretiers, soit au moins deux fois plus que le plus grand employeur connu en 1861. Quelques mois plus tard, un des meneurs des charretiers déclarera que :

There [are] at this moment six hundred men, formerly as happy as kings, some with their single horse and cart, who [are] now mere drivers for the Grand Trunk contractor. ${ }^{43}$

Ce chiffre nous semble nettement exagéré - il s'agirait de la moitié des charretiers de la ville à ce moment-là - mais la remarque reflète bien la perception qu'ont les charretiers de la menace que Shedden fait peser sur l'industrie du voiturage: la dépossession et la prolétarisation des charretiers indépendants, leur transformation en simples travailleurs.

\section{La grève de l'automne 1864 : les premiers jours}

En mai 1864 le Grand-Tronc amorce une nouvelle confrontation en renonçant unilatéralement à l'entente conclue l'automne précédent et en reprenant un tarif unique, global, comprenant le fret et le charroyage ${ }^{44}$. S'il ne semble pas y avoir de protestations ouvertes à ce moment-là, ni de la part des charretiers, ni de la part des marchands ${ }^{45}$, l'agitation reprend cependant au mois de septembre; cette fois, les charretiers, bien que mieux organisés et plus déterminés, font face à un adversaire intransigeant.

Lundi le 26 septembre et mardi le 27 , les charretiers se réunissent en assemblée en si grand nombre que beaucoup ne peuvent trouver de place dans la salle. Ils déclarent que malgré les requêtes

42 Voir des témoignages de marchands, Montreal Gazette (14 décembre 1865).

43 Montreal Herald (1er octobre 1864).

44 Montreal Gazette (3 octobre 1864).

45 Montreal Herald (1er octobre 1864). De plus, nous n'avons rien repéré dans les journaux à ce moment qui indiquerait des réactions de la part des charretiers. 
des charretiers, les marchands et la municipalité - qui ont pourtant leurs propres griefs envers le système de voiturage du Grand-Tronc - n'ont rien fait pour protéger les droits des charretiers face à Shedden et au Grand-Tronc. Ils décident donc de se mettre en grève pour obtenir l'abolition de la structure de tarifs uniques et l'abandon du système qui donne un monopole à Shedden. Ils demandent encore une fois la coopération des marchands, notamment dans des démarches auprès de la municipalité pour obtenir son intervention ${ }^{46}$.

Mercredi matin, les 1200 charretiers de Montréal sont en grève. Les rues et les quais sont déserts. Les charretiers se rassemblent sur les quais où des forces policières importantes maintiennent l'ordre. Malgré des déclarations assurant la protection policière pour ceux qui veulent travailler, et malgré les affirmations répétées quant à la reprise partielle du travail, le transport des marchandises reste toujours paralysé à travers la ville durant les trois jours suivants: «il était impossible de faire transporter quoi que ce soit» ${ }^{47}$; «the business streets have a kind of Sunday appearance. The vessels have had to ston working, and the port has a holiday ${ }^{48}$ ". Le steamer part pour Québec sans avoir été approvisionné. On parle de 2000 barils de farine perdus, et des milliers de livres sterling de marchandises abandonnées sans protection sur les quais ${ }^{49}$.

Ces premiers jours sont calmes - sauf de rares exceptions, personne n'essaie même de faire transporter quoi que ce soit. Tout le monde est plutôt occupé à se réunir, négocier et commenter la grève.

Les charretiers ont donc peu à faire pour assurer le respect de leur grève. Leurs propres rangs semblent solides - on n'entend pas parler de scabs. Ils se rassemblent sur les quais ou circulent par groupes de 12,15 , et 20 . Ils tiennent des réunions au moins une fois,

46 L'Union Nationale (27 septembre 1864 et 28 septembre 1864); Montreal Herald (28 septembre 1864). Pour ce qui suit - le déroulement de la grève, les réactions, etc. - nous nous sommes servis des récits des journaux à ce momentlà: le Montreal Herald, la Montreal Gazette, L'Union Nationale, Le Pays et, dans une moindre mesure, le Montreal Witness. D'autres journaux, comme le Montreal Transcript ou La Minerve, reprennent presqu'intégralement les comptes rendus d'un ou plusieurs de ces journaux, et n'ajoutent pas d'autres détails importants.

47 Le Pays (1 $1^{\mathrm{er}}$ octobre 1864).

48 The Montreal Witness (1 ${ }^{\mathrm{er}}$ octobre 1864$)$.

49 Montreal Herald (29 septembre 1864); Montreal Gazette (29 septembre 1864). 
quand ce n'est pas plusieurs fois par jour, où ils s'informent des derniers développements et réaffirment leur ligne de conduite. Médéric Lanctôt souligne dans un de ses articles le grand nombre de charretiers qui assistent à ces réunions, et le caractère démocratique $^{50}$ de celles-ci.

Ces réunions fréquentes sont un des deux éléments de base de l'organisation des charretiers, car à aucun moment on n'entend parler d'une association ou société quelconque, d'une structure plus permanente. Même au contraire, on entend Lanctôt souhaiter que les charretiers se dotent d'une véritable organisation:

... On ne saurait trop prêcher l'importance et l'utilité de l'organisation de nos différentes classes d'industrie. L'union et la cohésion communiquent la puissance. Espérons que les charretiers de la ville s'organiseront en société, comme leurs confrères d'autres industries. Leur intérêt l'exige et le commande. ${ }^{51}$

L'autre élément est un «comité de charretiers» qui assure la direction quotidienne de la grève, convoquant les assemblées et agissant comme porte-parole des charretiers auprès d'autres groupes. Bien que les origines et la base de ce comité soient inconnues, il semble jouir d'une certaine autorité ; ainsi, les porte-parole doivent s'y référer avant de prendre certaines décisions ${ }^{52}$. Cependant, c'est toujours l'assemblée qui décide en dernier lieu. La composition du comité, ou de ses porte-parole, varie quelque peu de jour en jour, mais certaines personnes s'y retrouvent toujours, à un titre ou un autre. Ainsi, on retrouve régulièrement James Curran, William Bennett, John et James Smith et William MacNeice, de même qu'A. Lecompte (probablement le fils de Toussaint). Plusieurs autres - Edward Dooner, Antoine Gauthier, J.-B. Renaud, entre autres - s'ajoutent au besoin ${ }^{53}$.

À l'exception de ce comité, il n'y a pas de véritable organisation pour assurer la continuité et le renforcement de la grève. On

so L'Union Nationale (4 octobre 1864).

51 L'Union Nationale (27 septembre 1864).

52 Montreal Herald (1 ${ }^{\text {er }}$ octobre 1864); voir aussi la lettre signée par Curran, « on behalf of Carters' Committee ", Montreal Gazette (3 octobre 1864).

53 Tous ces charretiers se retrouvent dans le recensement de 1861 , identifiés comme charretiers, sauf pour Curran et Bennett. Curran serait apparemment épicier à cette époque, tandis que Bennett est commis (travaillant pour le GTR, selon le Mackay's Montreal Directory, 1860-61). 
fait mention à plusieurs reprises d'un fonds de secours pour aider les charretiers les plus démunis à tenir le coup ${ }^{54}$, mais ce fonds n'aura guère le temps de se développer. Malgré ces faiblesses, durant la première semaine les charretiers croient fermement qu'ils pourront tenir aussi longtemps que nécessaire - jusqu'à l'hiver, selon l'un d'eux, tandis qu'un autre déclare qu'ils mangeront leurs chevaux avant de céder ${ }^{55}$.

\section{Les réactions à la grève}

Shedden, pour sa part, semble presque absent pendant la première moitié de la grève. Bien que son monopole soit l'enjeu principal de la grève, il n'intervient publiquement en aucun moment sur le fond du conflit. Il se contente de dire qu'il continue à fonctionner comme d'habitude et, à partir du lundi suivant, il tente d'agrandir son champ d'affaires. Son entreprise fait la plupart des rares tentatives de briser la grève.

C'est le Grand-Tronc qui répondra aux charretiers, mais seulement après plusieurs interventions de la part des marchands et de la municipalité (on prétexte l'absence de Brydges pour ne pas répondre, mais plusieurs charretiers affirment qu'il est toujours en ville, caché). Ce sont donc les marchands et, bien sûr, les journaux, qui réagissent le plus bruyamment pendant les premiers jours de la grève.

Parmi les journaux, le Montreal Herald se distingue par la quantité et la qualité soutenue de ses reportages. On y trouve de longs comptes rendus de toutes les assemblées principales des charretiers au cours de la grève, ainsi que des réunions publiques, qui frappent par leur ton plus objectif. En éditorial, le Montreal Herald met en doute tant la légalité que la légitimité du nouveau système du Grand-Tronc qui mène dans les faits à un monopole que la charte de la compagnie ne lui accorde nullement. Cependant, le journal, estimant que les charretiers auraient pu avoir un recours devant la loi, condamne leur choix de grève comme moyen de pression, et condamne, surtout, les quelques manifestations de violence.

Cette attitude est partagée par Le Pays, qui est cependant plus ouvertement favorable aux charretiers, estimant que leur cas est l'un

\footnotetext{
54 Voir, par exemple, l'offre de Lanctôt, L'Union Nationale (4 octobre 1864); aussi Montreal Herald (1 $1^{\text {er }}$ octobre 1864).

5s Montreal Herald (1 ${ }^{\mathrm{er}}$ octobre 1864); Montreal Gazette (3 octobre 1864).
} 
des rares où le recours à la grève serait presque justifié. De leur côté, le Montreal Witness et encore plus L'Union Nationale de Médéric Lanctôt appuient à fond la cause des charretiers, la reliant à d'autres griefs à l'endroit du Grand-Tronc, de sa position monopoliste et de ses amis politiques ${ }^{56}$.

La Montreal Gazette, par contre, se fait le grand défenseur de Shedden, de Brydges, du Grand-Tronc et du «progrès ». Le journal explique plusieurs fois les inconvénients de l'ancien système de voiturage pour le Grand-Tronc et justifie le nouveau dans les mêmes termes que Brydges: il rend plus efficace le fonctionnement des dépôts du Grand-Tronc et contribue ainsi à réduire les coûts et donc les tarifs; de plus, le monopole de Shedden ne fait que remplacer un patron par un autre, pour la plupart des charretiers, et finalement, ne change rien à leur statut. Au fond, il s'agit de l'avenir, du progrès, et on ne peut s'y opposer: "The strike is as reasonable, therefore, as that of hand-loom weavers against power looms, or seamstresses against sewing machines. ${ }^{57} \mathrm{Et}$, en parlant du progrès du chemin de fer qui réduit inévitablement le domaine du voiturage: "All regret the inconvenience to a class thus caused, but improvements cannot stop ${ }^{58}$.

Il accuse certains maîtres-charretiers jaloux de Shedden d'avoir entraîné les charretiers dans une grève qui ne concerne pas les véritables intérêts des charretiers, et déclare que d'autres journaux appuient et encouragent les charretiers par simple opportunisme politique. Soulignant les quelques incidents violents et le danger de «mob rule», il multiplie les avertissements contre la violence.

Les réactions des marchands et des représentants municipaux se précisent lors d'une série de réunions tenues le vendredi et le samedi.

56 Ils soulignent particulièrement la question de l'approvisionnement de Montréal en bois de chauffage, et le scandale autour d'un accident survenu récemment à Beloeil impliquant le Grand-Tronc, dans lequel un nombre important d'immigrants sont morts.

Médéric Lanctôt, rédacteur de L'Union Nationale et organisateur, quelques années plus tard, de la Grande Association de Protection des Ouvriers, joue un rôle particulièrement actif dans la grève, dénonçant lors d'une assemblée publique la conduite du Grand-Tronc, et adressant la parole à une assemblée de charretiers. Il offre aussi de contribuer à leur fonds de secours.

57 Montreal Gazette (30 septembre 1864).

58 Montreal Gazette (3 octobre 1864). 
Le vendredi matin, le Board of Trade convoque une réunion publique où le maire, des représentants des charretiers, le président du Board of Trade et plusieurs marchands prennent la parole ${ }^{59}$. Le maire, L. Renaud, est plus que sympathique à la cause des charretiers - il prend carrément position pour eux - et demande aux marchands de les soutenir s'ils veulent éviter un monopole dans le voiturage.

Quant aux marchands, pas un seul ne défend ouvertement la position du Grand-Tronc lors de ces réunions, bien que plusieurs témoigneront plus tard de leur satisfaction quant aux services de Shedden ${ }^{60}$.

Plusieurs d'entre eux appuient à fond les charretiers dans leurs griefs car ils sont eux-mêmes insatisfaits du nouveau système, soit à cause d'une opposition fondamentale aux monopoles (dont ils ne font pas partie), soit à cause de ses inconvénients (retards, inefficacité, coûts accrus de manutention dans les entrepôts). Pour ceuxci, il ne s'agit pas de négocier des modalités ou des structures de tarifs de voiturage du Grand-Tronc; ii faut tout simpiement refuser que le Grand-Tronc s'implique dans l'industrie du voiturage.

D'autres marchands - plus nombreux - se plaignent que les charretiers se sont attaqués à la mauvaise cible en faisant la grève, car ils ne nuisent qu'aux marchands qui sont de simples «tierces parties» innocentes, sans parti-pris ni pouvoir sur les événements. Ils sont toutefois prêts à intervenir auprès de Brydges pour obtenir des concessions pour les charretiers à condition que ces derniers acceptent de retourner au travail, ce qui est clairement le souci fondamental de la plupart des marchands. Ainsi, un comité est nommé pour rencontrer Brydges le lendemain, et en contre-partie Curran, un des principaux porte-parole des charretiers, accepte de recommander aux charretiers de reprendre l'ouvrage en attendant les résultats de cette intervention.

Les charretiers assistent nombreux à une assemblée le même soir où l'on donne un compte rendu de la réunion avec les marchands. Si Curran, fidèle à sa promesse, conseille aux charretiers de retourner au travail, les autres leaders ne sont pas du même avis

59 Pour le compte rendu de cette réunion, riche en détails, tant sur la grève que sur les attitudes et réactions des gens, voir le Montreal Herald $\left(1^{\mathrm{er}}\right.$ octobre 1864).

60 Montreal Gazette (14 décembre 1865). 
et l'assemblée finit par réaffirmer sa décision de rester en grève jusqu'à ce que des gestes concrets soient posés par les marchands.

Le lendemain le comité des marchands rencontre finalement Brydges, et lui présente deux demandes: que les tarifs de fret soient distincts des tarifs de voiturage; que les marchands puissent, s'ils le veulent, utiliser les charretiers de leur choix. Brydges rejette au départ le principe même de distinguer les tarifs de fret des tarifs de voiturage. Par contre, si les charretiers reprennent le travail immédiatement, il est prêt à revenir à l'entente de l'automne précédent, c'est-à-dire à des remises de $\$ 0.25$ à $\$ 0.35$ le tonneau pour les marchands utilisant leurs propres charretiers. De plus - concessions supplémentaires - il est prêt à garantir le maintien de l'entente pour une période de deux ans, et aussi d'examiner la possibilité d'ententes semblables pour les marchands recevant de grandes quantités de marchandises par chemin de fer à Montréal. Mais il refuse d'abandonner le principe d'une agence principale et privilégiée de voiturage (i.e. celle de Shedden), au nom de la plus grande efficacité et de l'économie du nouveau système et il continue à soutenir que celui-ci change peu de choses au fond pour les charretiers ordinaires.

Les marchands et le maire endossent cette nouvelle proposition de Brydges comme étant à la fois raisonnable et représentant le maximum de concessions auxquelles les charretiers peuvent s'attendre. Il la présentent aux charretiers et les encouragent à l'accepter et à retourner au travail le lundi matin. Curran l'endosse aussi comme la meilleure solution possible, quitte à contester devant les tribunaux le droit du Grand-Tronc de faire du voiturage. Cependant, malgré ses opinions personnelles, il est prêt à respecter la décision de la majorité.

Après deux réunions très militantes le samedi, les charretiers rejettent «presqu'unanimement» la proposition. Leurs raisons: l'entente ne leur donne pas l'égalité avec Shedden, car il subsiste toujours un écart de $\$ 0.15$ le tonneau dans les tarifs, et de ce fait elle ne constitue pas un "libre choix» pour les marchands; en fixant à $\$ 0.25$ et $\$ 0.35$ l'escompte donné aux marchands utilisant leurs propres charretiers, le Grand-Tronc fixe, en pratique, le tarif maximum que peuvent réclamer les charretiers pour ces trajets, mais ce tarif ne leur permet pas de survivre en tant que charretiers indépendants. Enfin, l'entente est d'une durée de seulement deux ans. Les charretiers rejettent donc tout compromis et se déclarent prêts à tenir jusqu'à l'hiver, s'il le faut. 
Ce refus qui survient le samedi soir constitue le sommet de la résistance des charretiers qui restent fermement sur leurs positions, malgré les avis contraires du maire, des marchands et d'au moins un de leurs leaders.

\section{Le règlement de la grève}

Leur détermination et leur solidarité ne sont cependant pas suffisantes pour maintenir un rapport de force qui leur soit favorable. $\grave{A}$ partir de ce moment, les charretiers deviennent de plus en plus isolés. Les appels à un retour au travail se font pressants, notamment dans les journaux. De plus, dès le lundi matin il y a des signes de nouveaux efforts pour assurer le transport des marchandises.

Shedden, en société avec un certain Thos. McCready, annonce qu'il est prêt à assurer le voiturage n'importe où dans la ville $^{61}$. Le Grand-Tronc annonce, par voie de la Montreal Gazette, son intention de fournir un nombre d'attelages suffisant pour le fonctionnement du port. En effet, il semble que certains travaux de charroyage souientit effectués au cours de la journée, soit par quelques charretiers indépendants, soit, dans la plupart des cas, par les charretiers du Grand-Tronc, c'est-à-dire de Shedden, ce qui mène au premier incident sérieux de la grève, quand un nombre indéterminé de charretiers attaquent des charretiers de Shedden le lundi soir. La Gazette laisse entendre aussi que d'autres travaux se font à la faveur de la nuit ${ }^{62}$.

Mais malgré ces efforts, la grève reste assez efficace, comme en témoignent les nouvelles commerciales. Pour dire le moins, les affaires de la ville continuent à tourner au ralenti :

At the Corn Exchange there was scarcely any business done this forenoon. The Carters' strike continued all day and everything wears an appearance of stagnation. ${ }^{63}$

The carters' strike continues we regret to say, and consequently the trade of the port is all but at a stand still. About forty or fifty barges laden with English and American coal brought via Quebec and Lake Champlain are lying idle with their cargoes on board. ... May other instances of the evil effects of the strike could be mentioned. ${ }^{64}$

$61 \quad$ Montreal Gazette (3 octobre 1864).

62 Montreal Gazette (4 octobre 1864), 2 et 3.

63 Montreal Gazette (3 octobre 1864).

64 Montreal Gazette (4 octobre 1864). 
Les journaux signalent la tentative de la part de plusieurs marchands influents de mettre sur pied une compagnie de voiturage qui serait indépendante et de Shedden et des charretiers réguliers tentative peut-être de bonne foi, mais dont on ne retrouve plus de traces une fois la grève terminée ${ }^{65}$.

Cependant, les négociations tournent aussi au ralenti. Après une réunion infructueuse avec George-Étienne Cartier, député de Montréal, procureur général et aussi avocat du Grand-Tronc, les charretiers semblent mettre tous leurs espoirs dans une intervention favorable du conseil municipal qui siège le mardi pour considérer la question.

Le mardi matin, le comité de charretiers présente une pétition demandant au conseil de:

...adopt such measures as will cause the freight and cartage to be separated, in order that the merchants and others requiring cartage may employ whomsoever they please. ${ }^{66}$

Les avocats de la ville donnent ensuite un avis juridique où ils affirment que le Grand-Tronc contrevient à sa charte en assurant un service de charroyage. D'après les avocats, il n'y a aucune raison de continuer la grève, puisqu'en soumettant leur cause aux tribunaux (ou même aux directeurs de la compagnie à Londres, suggèrentils !), les charretiers pourront obtenir justice. Après quelques débats, le conseil adopte ce rapport et nomme un comité spécial pour lui donner suite. Il demande aux charretiers de retourner au travail, en attendant la possibilité d'un recours légal. Le maire convoque alors ceux-ci et leur demande une réponse.

Deux des leaders, Bennett et Smith, déclarent qu'il faut se contenter de ce règlement et recommandent un retour au travail, en se fiant au maire et au conseil municipal pour obtenir justice. À quelqu'un qui demande des garanties, Bennett répond: "Can you expect more than this; you can't expect nor you can't get more. ${ }^{67}$

Sur cette note, les charretiers décident «unanimement " ${ }^{68}$ de reprendre le travail, et la grève est terminée.

65 Ibid; et Montreal Herald (4 octobre 1864).

66 Montreal Gazette (5 octobre 1864).

67 Ibid.

68 Le mot est du journal, ibid. 
Par contre, le conflit n'est pas réglé. Au cours de l'automne, la question revient devant le conseil municipal qui tente en vain de négocier avec Brydges pour les charretiers. Les charretiers se réunissent aussi à plusieurs reprises pour examiner divers moyens d'action. Au mois de janvier 1865, ils décident de porter l'affaire devant les tribunaux ${ }^{69}$. La cause traîne pendant toute l'année, et au mois de mai, moment du renouvellement des permis, les charretiers demandent au conseil municipal un «moratoire» sur les permis de Shedden jusqu'à ce que la cour ait statué ${ }^{70}$. Peine perdue, car Shedden obtiendra des permis pour 35 voitures à un cheval et 26 voitures lourdes à deux chevaux ${ }^{71}$.

Le juge Monk se prononce au mois de décembre 1865, et donne raison au Grand-Tronc. Si les charretiers perdent, dit Monk, c'est d'abord parce qu'ils ont mal formulé leurs demandes, et sur des points techniques de loi il doit donner raison à l'adversaire. Cependant, il y a aussi, et surtout, une raison de fond: les charretiers (et les marchands qui les appuient) n'ont pas réussi à prouver que le monopole du Grand-Tronc n'est pas dans les meilleurs intérêts du public. En accordant à Shedden des contrats privilégiés, sinon exclusifs, de voiturage, le Grand-Tronc ne fait que prendre les mesures nécessaires pour assurer la livraison en bon ordre du fret transporté par le chemin de fer. Il ne dépasse donc pas les limites de sa charte et ne cause pas de tort au public en général, au contraire ${ }^{72}$. Ainsi, la cour se range de l'avis du Grand-Tronc et accepte ses arguments d'efficacité et d'économie. Comme la Montreal Gazette le répétait, il s'agit du progrès, de l'avenir des transports. Et dans une cause où le procureur général était en même temps l'avocat de la compagnie accusée, il ne fallait pas s'attendre à ce que la cour mette des bâtons dans les roues du progrès, incarné pour l'occasion par le GrandTronc.

Les charretiers perdent donc leur grève. Mais même s'il a fallu attendre 15 mois pour en connaitre le dénouement final, la défaite décisive des charretiers se situe au moment de leur retour au travail, retour qui s'est effectué sans concession concrète (les offres de

69 Nous avons retrouvé des mentions de ces réunions surtout dans $L e$ Pays et L'Union Nationale, qui continuent à suivre la question plus fidèlement que les autres journaux.

70 L'Union Nationale (4 mai 1865).

71 Témoignage d'Olivier Bouchard, Montreal Gazette (14 décembre 1865).

72 Montreal Gazette (14 décembre 1865), 1 et 2. 
Brydges étant retirées après le refus des charretiers de reprendre le travail le samedi soir), sur la seule base de l'espoir d'une victoire juridique. Même si les raisons de ce retour au travail, qui constitue quand même un revirement assez soudain, peuvent sembler obscures, la défaite des charretiers était, d'une certaine façon, inévitable.

\section{Conclusion}

$\mathrm{Au}$ fond, les charretiers sont en grève contre la menace de la dépossession, de la prolétarisation. Ceux qui, comme Brydges ou la Montreal Gazette, affirment que la grève est uniquement une grève des maîtres-charretiers qui, jaloux de Shedden, y ont entraîné les autres pour protéger leurs propres intérêts, ont tort. Shedden constitue certainement un rival de taille pour les maitres-charretiers qui en sont sans doute jaloux. Mais Shedden représente aussi une menace bien précise pour la grande masse de charretiers indépendants, encore moins capables de soutenir la concurrence avec lui, et pour qui la marge entre le statut d'indépendant et celui de prolétaire est très mince. Pour ces charretiers, travailler pour Shedden signifie davantage qu'un changement de patron, car ils sont encore indépendants avec leur cheval et leur voiture; travailler pour Shedden, possédant lui-même chevaux et voitures, signifie se soumettre à un patron, bref, leur prolétarisation. Certes, Shedden est loin de contrôler l'ensemble du voiturage, mais les charretiers voient juste en percevant en lui une menace à leur avenir. Il est déjà, au moment de la grève, le plus grand employeur de charretiers et possède la plus grande concentration de chevaux et de voitures; dans les années qui suivent il étendra les ramifications de son agence, se lançant dans l'élevage de chevaux et créant ses propres ateliers de charrons et de forgerons ${ }^{73}$. Et si en 1864 c'est le transport intraurbain de marchandises qu'il vise, il s'engagera sept ans plus tard dans le transport de passagers, profitant d'une grève de cochers pour les concurrencer de la même façon qu'il a concurrencé les charretiers avec, encore une fois, l'appui du Grand-Tronc qui lui accordera une position privilégiée à ses gares ${ }^{74}$. Les charretiers indépendants ont donc bien raison de craindre la concurrence de

73 Montreal Directory, de John Lovell (1871). Shedden est encore aussi actif dans plusieurs autres domaines, dont la construction ferroviaire, les élévateurs à blé, etc.

74 Voir l'annonce de la Montreal Cab Com'y, Montreal Herald (6 mai 1871). 
Shedden, et ce sont leurs propres intérêts qu'ils défendent en se mettant en grève.

Les effets de la grève soulignent le rôle essentiel du voiturage à Montréal. Mais les réactions qu'elle provoque démontrent qu'en plus les charretiers ont su s'allier les marchands et la municipalité, dont certains intérêts sont aussi menacés et la susceptibilité parfois froissée par la puissance et l'arrogance du Grand-Tronc. Ils ont su aussi obtenir l'appui de plusieurs journaux qui, pour leurs propres raisons, s'opposent au Grand-Tronc ou à ses amis politiques. Il faut retenir, cependant, la nature limitée de ces appuis, qui ne se concrétisent que sous la pression des gestes des charretiers eux-mêmes. L'objectif premier de tous ces groupes ${ }^{75}$, et plus particulièrement des marchands, est avant tout de rétablir la circulation des marchandises; leurs propres griefs contre le Grand-Tronc, et encore plus ceux des charretiers, y sont vite subordonnés. Ils sont donc prêts aux compromis, et ne peuvent constituer un appui ferme et conséquent pour les charretiers.

Màis fondamentalement, la défaite des charretiers est déterminée par la taille et la force inégale des protagonistes principaux, qui découlent elles-mêmes du développement historique des transports, et qui rendent l'issue du conflit presque inévitable. La lutte oppose d'une part les charretiers indépendants et les petits maîtrescharretiers, petits artisans, travaillant de façon isolée, sans base réelle d'organisation commune, sans appuis conséquents et avec peu de ressources pour soutenir une longue résistance; et d'autre part, le Grand-Tronc, un monopole national du chemin de fer, avec des appuis politiques et financiers importants, pour qui Montréal - bien que dépôt important - ne constitue qu'une partie d'un réseau beaucoup plus vaste, et pour qui la situation dans le voiturage à Montréal constitue un obstacle à l'uniformisation de ses services dans les diverses villes qu'il dessert. Il s'agit effectivement d'une lutte entre les représentants des deux modes de production: le Grand-Tronc représente celui qui monte, le capitalisme industriel, tandis que les charretiers sont des artisans, voués à la marginalisation à long terme.

La Montreal Gazette a raison en disant que les charretiers luttent contre le progrès, mais il reste que leur lutte diffère quelque peu de celle de la plupart des autres artisans (des tisserands, par

75 Rappelons que L'Union Nationale fait exception ici. 
exemple) ${ }^{76}$. Dans ce conflit, ce ne sont ni leurs emplois, ni leurs outils de travail qui sont directement menacés; c'est plutôt la propriété de leurs outils qu'ils risquent de perdre. Ce sont leurs rapports de travail, et, finalement, leur survie en tant que classe qui sont en jeu. On peut donc comprendre leur détermination.

Mais leur situation d'artisans indépendants est à la source de faiblesses importantes. Nous avons remarqué le peu d'organisation chez les charretiers, mais il faudrait surtout souligner leur spontanéité et leur peu de stratégie. Les limites de leur stratégie amènent la défaite de leur grève, mais ces limites sont aussi peut-être inévitables. La grève, l'arrêt total de la circulation de marchandises, est leur seule arme, mais elle ne leur permet pas de s'attaquer directement au Grand-Tronc, leur adversaire principal qui, justement, les a déjà remplacés. Elle ne leur permet guère plus que de faire pression sur les marchands et le public pour que ces derniers fassent pression à leur tour sur le Grand-Tronc. Et ceci amène les charretiers, à chaque moment critique, à remettre leur sort entre les mains d'autres qui ne partagent pas fondamentalement les mêmes intérêts. Ainsi, ils font appel aux marchands, au Board of Trade, au conseil municipal et finalement aux tribunaux. C'est un cul-de-sac, car ces autres groupes n'agissent que sous la pression des charretiers qui, à long terme, ne peuvent maintenir un rapport de force favorable.

La lutte des charretiers rejoint celles de bien d'autres ouvriers à cette époque qui luttent contre le capitalisme montant, contre leur propre prolétarisation. L'intervention du Grand-Tronc accélère de façon très marquée ce développement, et provoque une confrontation ouverte, spectaculaire. Sa victoire est celle du capitalisme industriel. 